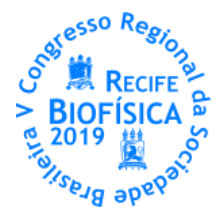

\title{
BIOPHYSICS OF THE RADIATION CYTOGENETIC DAMAGE
}

\author{
Thiago de Salazar e Fernandes ${ }^{1 *}$ \\ 'Departamento de Biofísica e Radiobiologia, UFPE \\ *thiagosalazar@hotmail.com
}

\section{INTRODUCTION}

Biodosimetry is a well stablished method for estimating absorbed dose of radiation in different scenarios, such as for patients undergoing radiotherapy, for the staff occupationally exposed to ionizing radiation, in cases of nuclear reactor accidents. This method is based on the scoring of dicentric chromosomes, rings and acentric fragments in peripheral blood lymphocytes, especially in cases when physical dosimetry was not performed. This allows the medical team to access the absorbed dose, helping the planning of therapy of such individuals. For doses higher than $1 \mathrm{~Gy}$, medical team can plan the therapy such as bone marrow transplant. For doses lower than $1 \mathrm{~Gy}$, doctors can estimate the risks of stochastic diseases, such as cancer. It is of great importance to standardize the protocols of biodosimetry. This can be done by the comparison between papers published in the specialized literature. However, these reports involve different scenarios, what cause a great variability and different interpretations among investigators, such as: What was the time passed between irradiation and blood collection? What was the scenario: chronic, protracted, acute or partial irradiation? What time Colcemid should be added during cell culture? Is there any biological approach that could make possible to detect the real fraction of cells exposed containing dicentrics? Is there a quicker and easy-to-perform method for screening radiation human exposures in mass casualties? There is no consensus yet for all these questions. There are many works in the scientific literature discussing most of these questions, and here it will be discussed the calculations of absorbed dose depending on the context of radiation exposure, and a possible methodological solution for some scenarios and screening, based on the understanding of the fractal high order chromatin organization.

\section{BIOPHYSICAL CONSIDERATIONS}

The importance of the mathematical method

The general formula to calculate absorbed dose in cases of acute exposures and of total body irradiation is:

$$
\begin{gathered}
Y=c+\alpha D+\beta D^{2} \\
\mathrm{D}=\frac{-\alpha \pm \sqrt{\alpha^{2}}-4 \beta(Y-c)}{2 \beta}
\end{gathered}
$$

Where $\alpha$ is the linear term, $B$ is the quadratic term, and $c$ is the frequency of dicentrics normaly found in non irradiated persons (named 'background'). At the same time, it is well known that most of the cases of human irradiation are not acute and the dose is not released for the total body of the exposed individual. In other words, this equation would give unreal estimations in cases of:

1. Partial body exposures;

2. Chronic exposures (occupational exposures);

3. Protracted exposures;

4. Fractionated exposures.

Therefore, it must be taken into account the context of exposure, to use the equation (linear or quadratic). One example is the case of protracted exposures. Lea and Catcheside (1942) (apud IAEA, 2001; 2011) have demonstrated that it is needed for the better estimation of dose to include another function, $G(x)$ function, multiplying the beta term, which considerer the ' $\mathrm{t}$ ', which is the overall time that the individual was exposed, and ' $t 0$ ', which is the time of DNA repair (around 2 hours). Therefore, if the irradiation takes place in a time longer than 15 minutes, the quadratic equation is transformed to considerer the $G(x)$. The $G(x)$ is igual to:

$$
Y=c+\alpha D+\beta G(x) D^{2}
$$

Where

$$
G(x)=\frac{2}{x^{2}}\left(x-1+e^{-x}\right) ; x=\frac{t}{t_{0}}
$$

Another context is whether the radiation exposure was chronic. Now again, it is necessary the transformation of the general equation. In this case, it is used the linear coefficient, because the quadratic component of the equation is too low because of the time interval between one track of radiation and the next, becoming the beta term negligible, shown below:

$$
Y=c+\alpha D
$$

(5)

For a better approximation, according to Braselmann et al. (1994), we would need to include a $\mathrm{G}(\mathrm{x})$ function (modified by the present work) multiplying the alpha coefficient, that take into account the time of lymphocyte renew and the time of the formation of new (or 'de novo') chromosome aberrations. This is done by the integration of dose in time, resulting in the following function:

$$
Y=c+\alpha G(x) D
$$

$$
G(x)=\frac{1}{x}\left(1-e^{-x}\right) ; x=\frac{t}{t_{0}}
$$

Where $\mathrm{t}$ is the time of work, and to is the mean life-time of lymphocytes.

This mathematical correction is of great importance for not allowing the underestimation of absorbed dose in cases of chronic exposures. 
It is important to emphasize that most of the human exposures to ionizing radiation does not involve the total body irradiation, being partial body irradiations (e.g., arms, legs, head, chest). As a consequence, the estimation of absorbed dose by scoring dicentrics in peripheral lymphocytes can result in underestimations if the laboratory does not considerer the complexity of the scenario (LLOYD et al., 1991; PRASANNA et al., 2005).

This is due to the fact that the lymphocytes are circulating cells. Thus, those cells not belonging to the irradiated area of the body will be also present in certain amount in the blood samples. This will lead to a decrease of the total frequency of dicentrics, and consequently, to an underestimation of the absorbed dose (IAEA, 2001; 2011).

To correlate the frequency of chromosomal aberrations with the dose to the part of the body irradiated, one must considerer a period of $24 \mathrm{~h}$ for irradiated lymphocytes being redistributed in peripheral blood (IAEA, 2001). Taking into account a homogeneous distribution of blood in the human body, it can be inferred that 24 $\mathrm{h}$ after irradiation the frequency of unstable chromosome aberrations in peripheral blood will be proportional to the percentage of the patient's irradiated volume. According to the data from the planning of dose distributions in cases of cervical cancer, the irradiated volume of each patient corresponded to about $1 / 5$ of the total volume of their bodies (SILVA et al., 2005). To solve eventual mistakes in dose estimates, it has been proposed mathematical methods such as the CP method (Contaminated Poisson), that is considered to the irradiated fraction of the body, and the Qdr method, which considerer only the cells containing dicentrics plus rings and assumes that these cells were exposed in the moment of the irradiation (SASAKI; MIYATA, 1968).

According to the $\mathrm{CP}$ method, the cells that contain chromosome aberrations belong to the irradiated region of the body, while normal cells not damaged belong to two subpopulations: (a) those belonging to the non irradiated fraction of the body and (b) those irradiated, representing the term $\left(\mathrm{e}^{-\mathrm{a}}\right)$ from Poisson distribution (IAEA, 2001; 2011).

The mathematical correction of the yield of dicentrics $(\mathrm{Y})$ by the $\mathrm{CP}$ method, and the obtention of the irradiated fraction ( $\mathrm{f}$ ), can be calculated by the equations (8) and (9):

$$
\begin{gathered}
\frac{Y}{1-e^{-Y}}=\frac{X}{N-n_{0}} \\
Y f=\frac{X}{N}
\end{gathered}
$$

Where $\mathrm{N}$ is the total number of cells, $\mathrm{X}$ is the number of dicentrics and $n_{0}$ is the number of cells without dicentrics.

The mean dose can be obtained applying the $Y$ value obtained by Equation (9) in the quadratic equation (Equation 1). The fraction of the body irradiated can be estimated after correction considering the effects of cell death (apoptosis) and mitotic delay. This last makes mention to the delay that irradiated cells have in reaching metaphase in conventional cell cultures of 48 hours. If the fraction of irradiated cells which reach metaphase is $p$, the fraction of the body irradiated $(\mathrm{F})$ can be obtained by the Equation 10 (IAEA, 2001; 2011):

$$
F=\frac{f}{(f+p)-(f * p)}
$$

The $p$ value is used to correct the value of $f$, considering the mitotic delay and apoptosis, because not all cells survive to reach metaphase. The same is obtained with the dose that reduces in
$37 \%$ the number of viable cells (i.e., $D_{0}$ ). For example, if the $p$ value is 0.33 , this means that only $33 \%$ of the cells are capable to reach metaphase after 48 hours cell culture. Thus, using this value in Equation 10, the value of cells counted is adjusted to provide the real fraction of the body which was irradiated (F) (IAEA, 2001; 2011).

The Qdr method, on the other hand, avoids problems of dilution of the irradiated cells with those non belonging to the exposed area, or the renew of cells from the diferentiation of stem-cells from bone marrow. With this, Qdr is the expected production of dicentrics, $\mathrm{X}$, among those cells presenting aberrations, $\mathrm{Nu}$, and can be calculated by Equation 11 .

$$
Q d r=\frac{X}{N_{u}}=\frac{Y}{1-\mathrm{e}^{-\left(Y_{1}+Y_{2}\right)}}
$$

Where $\mathrm{Y} 1$ and $\mathrm{Y} 2$ are the production of dicentrics plus rings and the excess of fragments, respectively. As $\mathrm{Y} 1$ and $\mathrm{Y} 2$ are known functions of dose and are derived from the calibration doseresponse curve obtained by the in vitro irradiation of peripheral lymphocytes, Qdr is a function of dose alone and allows to estimate the dose for the fraction of the body which was irradiated.

\section{Can mathematical corrections be replaced by biological methods?}

We saw before how mathematical equations can simulate most of the biological dosimetry scenarios. The question presented is whether the dose could be calculated with no need of mathematical corrections, by prolonging the time of cell culture in order to detect the fraction of irradiated cells. If "yes", no need for mathematical corrections would be necessary.

This hypothesis was raised because considering that the cells which were in the part of the body irradiated would be in 'mitotic delay', and when we mixture these cells with the non irradiated cells, it is expected that the irradiated fraction due to the stops induced by 'checkpoints' during cell cycle will delay the cells to divide. If we give more time for the irradiated cells to reach metaphase, this will allow those cells under microscope. Therefore, this is what happen after cell cultures of 72 hours, putting Colcemid at the beginning of culturing. The fraction of irradiated cells start to be seen more and more, in first divisions (M1). At this total time, unirradiated cells will be at second or more divisions, and irradiated cells will be reaching the first divison, what can be distinguish by the FPG (Fluorescence Plus Giemsa) method.

When the quadratic equation is used considering only the M1 cells in 72 hours cell cultures, the absorbed dose will be naturally corrected, with no need for mathematical corrections.

It is, of course, a bit pretentious that this simple method would solve all the problems of partial body exposure. In fact, most of the studies are limited to in vitro simulations, and more data of in vivo investigations are needed (FERNANDES et al., 2008).

\section{Global architecture of chromatin: fractal analysis}

The main drawback of cytogenetic dosimetry continues to be the time consuming of cell culturing and the laborious analysis of all 46 chromosomes per metaphase. Studies that investigate cells in interphase are still scarse, and are limited nowadays to only one technique, the premature chromosome condensation (PCC).

More recently, the global architecture of the chromatin was analysed by fractal analysis (XAVIER et al., 2018). The idea of this research was based on the fractal globule model, by LiebermanAiden (2009). Fractal is an object with a non entire dimension, with self-similarity and with scale invariance. This is all presented 
in chromatin condensation, which follows a mathematical model so-called 'peano curve'. The fractal dimension can be measured but a method called box-counting, as shown in equation 12 :

$$
D F=\lim _{r \rightarrow 0}\left(\frac{\log N r}{\log 1 / r}\right)
$$

Where $\mathrm{Nr}$ is the number of boxes necessary to cover the image in each progressive reduction of the side of the box ( $r$ ). FD will be the slope of the regression line, generated by the logarithm of the quantity of boxes in function to the logarithm of the size of the boxes.

By the measurement of fractal dimension of the chromatin of irradiated lymphocytes, compared to non-irradiated lymphocytes, it as possible to distinguish between irradiated and non-irradiated sample (XAVIER et al., 2018). This may be an alternative method for screening irradiated victms in cases of mass casualties, where a high number of individuals has to be evaluated in a short period of time. This method is quick and easy-to-perform, because does not need cell culture or chromomal analysis, and may be performed using a 'friendly' computational platform, such as the free software Image J, from the National Institute of Health $(\mathrm{NIH})$, United States.

\section{FINAL CONSIDERATIONS}

In this review, it was presented a repertoire of mathematical methods (linear and non-linear) for calculating absorbed dose and identifying individuals exposed to ionizing radiation. One of the major confounding factors is what equation to use for estimating the absorbed dose in different scenarios, such as protracted irradiations, chronic exposures, fractionated irradiations and partial body irradiations. The simplest way is to considerer the suspected cases as uniform exposure. However, for better estimations, mathematical corrections are inevitable in most of the cases. On the other hand, not only mathematical corrections are possible, and a methodological adaptation such as prolonging the time of cell culture was presented. The analysis of global chromatin architecture by fractal dimension measurement is also a quick and easy-to-perform alternative for human radiation exposure screening, due to the no need for cell culture and chromosomal analysis.

\section{REFERENCES}

BRASELMANN, H.; SCHIMID, E.; BAUCHINGER, M. Chromosome aberrations in nuclear power plant workers: the influence of dose accumulation and lymphocyte lifetime. Mutation Research, v. 306, p. 197-202, 1994.

FERNANDES, T. S.; LLOYD, D.; AMARAL, A. Biodosimetry for dose assessment of partial-body exposures: a methodological improvement. Brazilian Archives of Biology and Technology, v. 51, special number, p. 97-102, 2008.

FERNANDES, T. S.; SILVA, I. M. S.; MORAES, R. B.; AGUIAR, L. A. A.; AMARAL, A.; BARBOSA, C. T.; NOGUEIRA, R. A. Non-linear dynamics of chromosome condensation induced by Colcemid. Brazilian Archives of Biology and Technology, v. 56, n. 1, p. 85-92, 2013. INTERNATIONAL ATOMIC ENERGY AGENCY (IAEA). Cytogenetic analysis for radiation dose assessment. Technical report series. Vienna, n. 405, 2001.

INTERNATIONAL ATOMIC ENERGY AGENCY. laea Annual Report 2011 [Internet]. Vienna, Austria; 2011.

LIEBERMAN-AIDEN, E.; BERKUM, N. L.; WILLIAM, L.; IMAKAEV, M.; RAGOCZY, T.; TELLING, A.; et al. Comprehensive mapping of longrange interactions reveals folding principles of the human genome. Science, v. 326, p. 289-293, 2009.
LLOYD, D. C.; EDWARDS, A. A.; NATARAJAN, A. T.; DARROUDI, F. Biological dosimetry applied to in vitro simulated partial body irradiation. In: Treatment and Biological Dosimetry of Exposed Persons: Post-Chernobyl Action (K. Chadwick and G. Gerber, Eds.), p. 51-80. Report No. EUR 12558, Commission of the European Communities, Luxembourg, 1991.

PRASANNA, P. G. S.; MARTIN, P. R.; SUBRAMANIAN, U.; BERDYCHEVSKI, R.; KRASNOPOLSKY, K.; DUFFY, K. L.; MANGLAPUS, G. L.; LANDAUER, M. R.; SRINIVASAN, V.; BOREHAM, D.; HAGAN, M. P.; JINARATANA, V.; BLAKELY, W. F. Cytogenetic biodosimetry for radiation disasters: Recent advances. Bethesda, MD, USA: NATO RTG-099, 2005.

SASAKI, M. S.; MIYATA, H. Biological dosimetry in atom bomb survivors. Nature (London), v. 220, p. 1189-1193, 1968.

SILVA, I. M. S.; MAGNATA, S. P.; AMARAL, A.; SOTERO, G.; CAVALCANTI-MELO, $\mathrm{H}$. Dose assessment by quantification of chromosome aberrations and micronuclei in peripheral blood lymphocytes from patients exposed to gamma radiation. Genetics and Molecular Biology, v. 28, p. 452-457, 2005.

XAVIER, A. I. S. F.; CAVALCANTI, M. B.; AMARAL, A.; FERNANDES, T. S. Fractal analysis of chromatin as a potential indicator of human exposure to ionizing radiation. Scientia Plena, v. 14, n. 2, p. 1-8, 2018. 